\title{
Developing and Evaluating Mobile Sensing for Smart Home Control
}

\author{
Rischan Mafrur, Priagung Khusumanegara, Gi Hyun Bang, Do Kyeong Lee, I Gde \\ Dharma Nugraha and Deokjai Choi \\ School of Electronics and Computer Engineering, Chonnam National University \\ Gwangju, South Korea \\ \{rischanlab,priagung.123\}@gmail.com,badmanner@naver.com, \\ ldk7175@nate.com,gdebig@gmail.com,dchoi@jnu.ac.kr
}

\begin{abstract}
Many of researches in controlling smart home system have been proposed. Most of previous approaches in controlling smart home system requires interventions and commands from user. This paper propose a system about smart home based on mobile sensing that does not requires interventions and commands from the user. Mobile Sensing is used to records daily routine activities of the user. Then the system automatically gives a response to user based on his/her daily routine activities. We have implemented our approach to demonstrate the feasibility and effectiveness of using mobile sensing for controlling smart home system. Furthermore, we evaluate our approach and present the details in this paper.
\end{abstract}

Keywords: Smart home, mobile sensing, ubiquitous sensors

\section{Introduction}

Many approaches have been addressed in smart home field, especially for smart home control. Common approaches for controlling smart home uses a device as an interface between users and the system. The example of the device such as phone, tablet, and PC. Furthermore, the concept of internet of things make device provider to develop smart devices to support smart home system. Nowadays, we can find many systems that uses smart devices in market such as smart air conditioning system, smart light system, and etc. Those smart systems can gives a response based on human existence or environmental condition. On the other side, mobile phones are rapidly becoming popular in people's lives. Today's mobile phones come with a set of powerful embedded sensors, such as an accelerometer, magnetic field, gyroscope, GPS, microphone, and camera. Those sensors could be used for many purposes.

Our research focus on developing smart home system which automatically gives responses based on user's daily activity. These systems enact user daily routine activity as the knowledge base for giving the response to the user. To record user's daily activity, we utilize the sensors in mobile phone. The sensors that we used are GPS, accelerometer, and magnetic field. Example scenario of user's routine daily activity: Alice is a student researcher in one of university in Korea. Every working day, his activities are wakes up in the morning, takes a shower, get breakfast, and goes to his campus at 8:40 AM. He is living in dormitory, so he walks from dormitory to his lab (campus) and takes time about 10 minutes. Usually, he arrives in his lab at $9 \mathrm{AM}$ and then sits on his chair and starts working. This example is one of the human daily routine in working day. Based on this example scenario, we can used GPS to determine Alice's location, whether he is in dormitory or in his lab. But, GPS cannot be used for detecting human location inside the building. The solution is using magnetic field sensors 
to determine user position inside the building [33-36]. To know his activities such as walking, running, standing, walking down stairs, or sitting, we use accelerometer sensors [22-32]. Based on sensor data that we collected from users, we determine their daily routine and then using this knowledge to develop our smart home system.

In this research, we divide the architecture into two systems. First is a server that running as a system for monitoring and command receiver. Second is a client that running as a control system. Servers consist of Raspberry Pi for the hardware and Heimcontrol.js for the home automation library. We use Raspberry Pi because easy to modify, flexible and open source. We use Heimcontrol.js ${ }^{1}$ library in server because it is the one of awesome home automation library which full support with Raspberry Pi and Arduino. This library use Node.js, Mongo DB as databases, HTML5 and Websockets. We use android devices for the client and we modify the Heimdroid ${ }^{2}$ application as client application. Actually, this paper is improvement from our previous work [2]. In the previous work, we can achieve $88 \%$ accuracy (correct responses) in real environment but we have many of delay problems. In this research, we tried to solve that problem and proposed a new approach which has better performance.

The rest of this paper organized in to six sections. Section 2 discusses about state of the art, the related previous works. In section 3 we presents about our system architecture and scenario. In section 4, we explain about methods that we used in our proposed smart home system. In section 6, we presents about the result and analysis of our approach. Section 7 is the conclusion of this paper.

\section{State of Art}

\subsection{Smart Home}

Most of researches in smart home area are divided into three parts: smart home design, authentication and security, and human interaction. In smart home design, the research focus on optimum design for communication and low power [1, 3-12]. In authentication and security, the researches focus on utilization of PIN and biometrics [13-16]. And in human interaction, the research focus on develop the interface between user and smart home system. The research develop the interface that utilize application such us android client application or voice command [17-21].

The definition of Smart Home System was proposed in [11]. [11] defined that smart home system should have 6 main components such as efficient automation of routine task, security of automation systems, easy in use, local and remote access, tele-monitoring, system cost and flexibility. Smart home system also composed by many sub system. Researchers in [6] propose multi agent based on intelligent sensor and actuator network for smart home. They build simulation which provide multi agent design framework. They proposed BDI model (Belief, Desire, and Intention) for every agent individual behavior design.

Many of ideas have been proposed on how to design efficient system that always consider about communication among devices, saves energy and etc., [3] propose smart home design using WSN. The designs consist of Microchip PIC16F877A MCU as the main component and supported by Zigbee and sensors. This paper shows that by using WSN they can detect, monitoring and controlling fire, gas leakage, water leakage, intruder, temperature, and illumination. Many of papers research also focus on low power (energy optimization) in smart home. Most of the proposed smart home system was using low power protocol ZigBee [4, 9], Modbus [12], and Hydra Framework [10]. In [7] present the design of smart home

\footnotetext{
${ }^{1}$ https://ni-c.github.io/heimcontrol.js/

${ }^{2}$ https://github.com/JorisM/Heimdroid
} 
system based on 6LowPAN WSN. In [8] explain the details about the disadvantages of IP technology for smart home system. Some of the disadvantages of IP Technology are heavy weight and expensive. Our previous research also consider the disadvantages of IP Technology and have develop smart system home [1] based on battery less 6LowPAN. The smart home system was developing not only based on 6LowPAN, but also consider about energy harvesting.

In smart home and human interaction area, many ideas have been proposed. In [19] propose user friendly interface for smart home and using Bluetooth as media communication between server (controller, in this case is PC) and client. Similar work by [20], but the proposed system use Arduino BT board. The similarity of the proposed system so far are users have to interact with application GUI in their mobile phone and they have to turn on the Bluetooth to connect with the system. Another way proposed by [21] that used Raspberry Pi for the HAS server and the email as the media communication to the users. Researchers in [21] developed some algorithm inside the smart home for receiving and reading email and then send the responds with corresponding instructions. In [17] propose the smart home system with interaction between smart home system and user using low cost touch screen technology. Furthermore, [18] propose the system that use voice commands as the media communication between user and smart home system. The proposed system was called PATSH that performs real time recognition of voice commends for controlling the system.

The last area in smart home system research but also has important role is authentication and security. The researchers in [13] propose the design of smart home with security system using android ADK. They claim that with android ADK they can develop authorized system with low cost, scalable for modification, and the system can control smart home easily using android phone or tablet. Moreover, biometrics for authentication in smart home area also has been proposed by many researchers. In [16] propose the system using biometrics ear shape for smart home authentication and utilize front camera in smart phone to getting ear shape photo. In security area, there are many ideas about security monitoring in smart home. Paper [14] introduce the development of smart home control and security system based on using field programmable gate array (FPGA) and web technology. The user can receive the security information and feedback from system via email or SMS. Another paper [15] propose integration security monitoring system for smart home by using video camera that automatically recording when motion is detected and reported to user via mobile phone.

\subsection{Smartphone Sensing}

Mobile sensing that embedded in mobile phone have been exploited by the researcher for many useful system. For example develop activity recognition system that utilize accelerometer sensor, health care monitoring system that utilize a set of sensors in android phone, recommendation and user moving prediction system that utilize accelerometer, GPS and magnetic sensor, and many other system. In this section we describe three sensors that have been used for previous system that have correlation with our present research: accelerometer sensor for user identification, accelerometer sensor for activity recognition, and magnetic field sensor for determine indoor user position.

\subsubsection{Accelerometer for user Identification and Activity Recognition}

Most of previous researches utilize this sensor for user identification based on user's gait pattern. In this research, we exploit this sensor for two of purposes: user identification and activity recognition. We summarize from previous work [22-28] which use accelerometer for user identification in Table 1. Not only for user identification we can use this sensor for 
recognize user activity [29-32] such as standing, walking, running, jumping, sitting, walking upstairs and downstairs. Table 2 shows the previous work which use accelerometer for activity recognition. From Table 1 and 2 we can see that both system, user identification and activity recognition, have good result.

Table 1. User Identification based on Accelerometer Data [Previous Work], SVM (Support Vector Machine), HMM (Hidden Markov Model), DTW (Dynamic Time Wrapping), CWT (Continuous Wavelet Transform)

\begin{tabular}{|c|c|c|c|c|c|}
\hline Previous work & Sampling Rate & $\begin{array}{c}\text { Devices } \\
\text { Location }\end{array}$ & Method & $\begin{array}{l}\text { Number of } \\
\text { Subject }\end{array}$ & Result \\
\hline$[22]$ & - & $\begin{array}{c}\text { Pocket \& } \\
\text { Hand }\end{array}$ & SVM & 30 & $\begin{array}{c}\text { more than } 60 \% \\
\text { accuracy }\end{array}$ \\
\hline [23] & $10 \mathrm{~Hz}, 100 \mathrm{~Hz}$ & Pocket & SVM, CWT & 36 & $\begin{array}{l}99.4 \% \text { VR, } \\
0.1 \% \text { FAR }\end{array}$ \\
\hline [24] & $25 \mathrm{~Hz}$ & Hip & HMM & 48 & $6.15 \%$ EER \\
\hline [25] & $37 \mathrm{~Hz}$ & Hip & SVM & 6 & $\begin{array}{c}90.3 \% 3.2 \% \\
\text { RR }\end{array}$ \\
\hline [26] & $45 \mathrm{~Hz}$ & Hip & DTW & 51 & $20 \%$ ERR \\
\hline [27] & $25 \mathrm{~Hz}$ & $\mathrm{~T}$ pocket & SVM & 25 & $100 \%$ RR \\
\hline [28] & $120 \mathrm{~Hz}$ & $\mathrm{~T}$ pocket & HMM & 48 & $\begin{array}{ll}6.15 \% & \text { ERR, } \\
5.9 \% & \text { FMR, } \\
6.3 \% \text { FNMR }\end{array}$ \\
\hline
\end{tabular}

Table 2. Activity Recognition based on Accelerometer Data [previous work], SVM (Support Vector Machine), NB (Naive Bayes), DT (Decision Tree), KDA (Kernel Discriminant Analysis)

\begin{tabular}{|c|c|c|c|c|c|c|}
\hline $\begin{array}{l}\text { Previous } \\
\text { work }\end{array}$ & $\begin{array}{c}\text { Sampling } \\
\text { Rate }\end{array}$ & $\begin{array}{c}\text { Device } \\
\text { Location }\end{array}$ & Method & $\begin{array}{l}\text { Number of } \\
\text { Subject }\end{array}$ & $\begin{array}{c}\text { Number of } \\
\text { Activity }\end{array}$ & Result \\
\hline [29] & $20 \mathrm{~Hz}$ & pant pocket & $\begin{array}{l}\text { hierarchical } \\
\text { clustering }\end{array}$ & - & 5 & $\begin{array}{l}\text { more than } \\
90 \% \text { accu- } \\
\text { racy }\end{array}$ \\
\hline [30] & $\begin{array}{l}20 \mathrm{~Hz}, 100 \\
\mathrm{~Hz}\end{array}$ & $\begin{array}{l}\text { hip,wrist, arm, } \\
\text { ankle and thigh }\end{array}$ & $\begin{array}{l}\text { SVM, } \\
\text { NB,DT }\end{array}$ & 20 & 5 & $\begin{array}{l}\text { more than } \\
95 \% \text { in NB and } \\
\text { SVM and close } \\
\text { to } 100 \% \text { in DT }\end{array}$ \\
\hline $\begin{array}{l}{[31]} \\
{[32]}\end{array}$ & $20 \mathrm{~Hz}$ & $\begin{array}{l}5 \text { different } \\
\text { position }\end{array}$ & $\begin{array}{l}\text { KDA-Based } \\
\text { SVMs }\end{array}$ & 30 & 15 & $\begin{array}{l}\text { average } \\
99.1 \%\end{array}$ \\
\hline & - & $\begin{array}{l}\text { right palm of } \\
\text { the hand with } \\
\text { the screen } \\
\text { faced upwards }\end{array}$ & Naive Bayes & 1 & 5 & $\begin{array}{l}\text { accuracy } \\
\text { accuracy } \\
92 \%\end{array}$ \\
\hline
\end{tabular}

Table 3. Indoor Positioning with Magnetic Field Sensor [Previous Work]

\begin{tabular}{|c|c|c|c|c|c|}
\hline Previous work & \multicolumn{1}{|c|}{$\begin{array}{c}\text { Device } \\
\text { Position }\end{array}$} & \multicolumn{1}{|c|}{ Devices } & $\begin{array}{c}\text { Device } \\
\text { Location }\end{array}$ & Methods & Result \\
\hline$[33]$ & - & $\begin{array}{l}\text { Acer A500 } \\
\text { tablet }\end{array}$ & $\begin{array}{l}\text { 4different lo- } \\
\text { cation (home) }\end{array}$ & FFT & $0 \%$ error \\
{$[34]$} & - & Atmega128 & $\begin{array}{l}\text { Hallway } \\
\text { in front of }\end{array}$ & $\begin{array}{l}\text { Simple path al- } \\
\text { gorithm }\end{array}$ & $\begin{array}{l}\text { Average error } \\
0.425 \mathrm{~m}\end{array}$
\end{tabular}




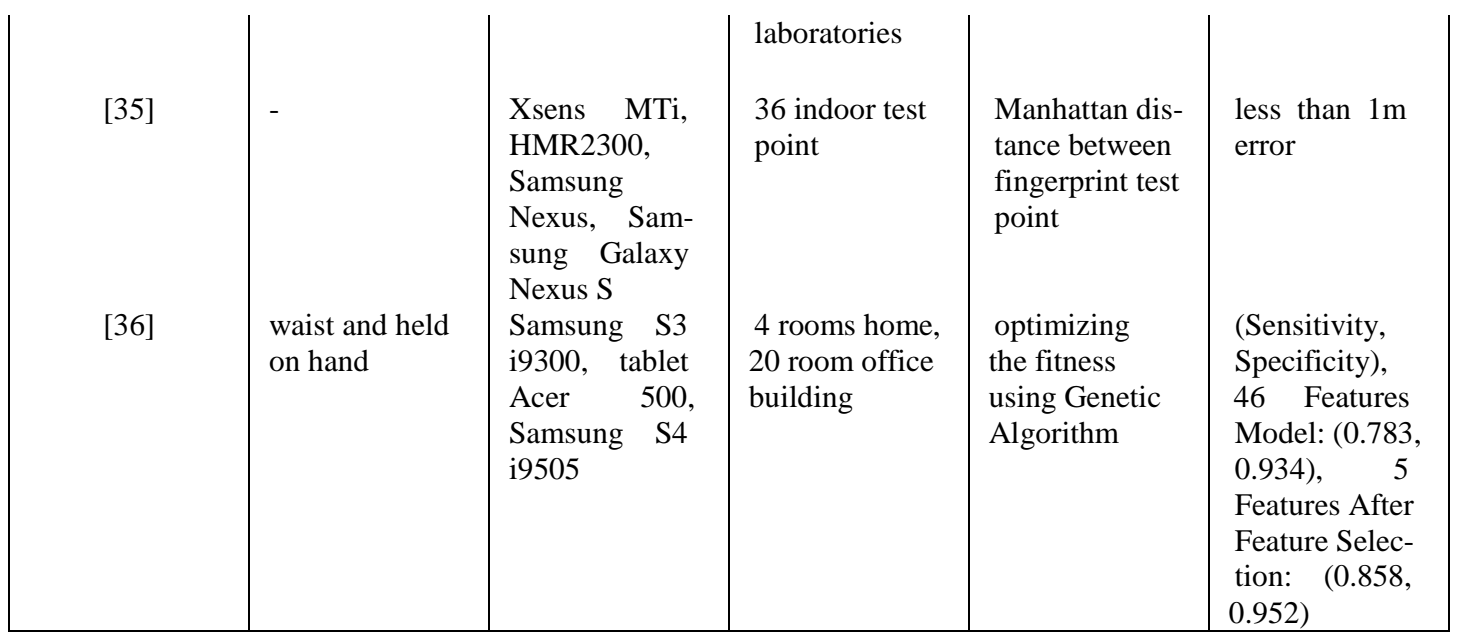

\subsubsection{Magnetic Field for Determine Indoor user Location}

Indoor Positioning System (IPS) is the one of hottest research topics. Many of papers have been discussed about this. One of the approaches to determine indoor position is using Wi-Fi. One of example was [37], they present indoor positioning method based on Wi-Fi fingerprints especially using RSSI measurements from multiple Wi-Fi access point. But using Wi-Fi for IPS has many limitations. Even though Wi-Fi RSSI signatures are available in indoors but it's vary significantly over time and susceptible to human presence. In [38] tried to overcome these limitations by using FM broadcast radio for indoor fingerprinting. FM signals has lower frequency than Wi-Fi so it less susceptible to human presence.

Location of Wi-Fi AP that have no guarantee always same over time, become another limitation of Wi-Fi. Sometimes there is any possibility that someone move the APs to others place because it easy to be moved. Moreover, the used of Wi-Fi will increase the smart phone power consumption so it is not good from user viewpoint. In [38] mentioned that the power consumption for using Wi-Fi module is $300 \mathrm{~mW}$ in idle power-saving mode and it will drains the phone's battery life quickly. To overcome the limitation of Wi-Fi module, many discussion propose magnetic field or magnetometer sensor in android as the solution.

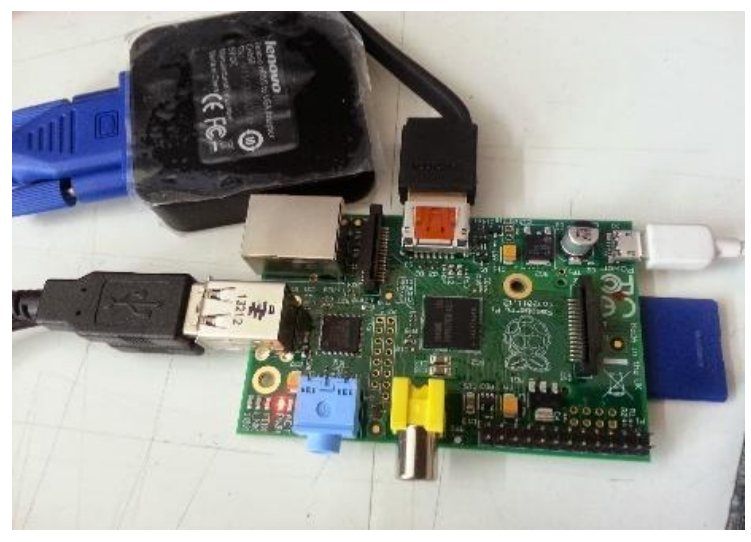

Figure 1. Raspberry Pi

The ideas to develop IPS using magnetic field have been proposed by many papers. In [37] tried to implement magnetic field sensor for location identification. These papers [37] use a house which has 4 rooms (living room, dining room, kitchen and bathroom) as a test bed. 
Then exploit magnetic field sensor to identify each location. This paper implement the approach using Acer A500 tablet is used for a client and FFT (Fast Fourier transform) to analyze the data. This paper's approaches have result $0 \%$ error. Similar research in [34] was doing experiment walking in hallway in the front of laboratories and the average error result was $0.425 \mathrm{~m}$, in [35] using 36 indoor test point with 4 kind different devices and the average error was less than $1 \mathrm{~m}$. In [36], the experiment was more complex. This paper use 4 rooms in home and 20 rooms office building. This paper present the genetic algorithm for optimizing the fitness and the result of the experiment is divided into two result. First result, using all the features model (46 features) generate 0.783 sensitivity and 0.934 specificity. Second result, using 5 features after features selection generate better result that 0.858 sensitivity and 0.952 specificity.

The real IPS application has created by researchers from University of Oulu in Finland which called IndoorAtlas ${ }^{3}$. They were doing similar work using magnetic field for IPS. They said every square inch of Earth emits magnetic field and then this field modulated by concrete and steel structures which made by human. This system has an accuracy between 0.1 and 2 meters. Table 3 summarizes indoor positioning system approaches by using the magnetic field sensor.

\section{Architecture and Scenario of Experiments}

In this section, we have two subsections. First subsection, we explain about our system architecture that consist of server side and client side. Second subsection, we explain about the scenario of our experiment.

\subsection{System Architecture}

In the context of smart home, we have to consider about energy saving and lightweight processing. We did not use big server with big processing. In this research we use Raspberry $\mathrm{Pi}$ as our server because open source, flexible, and easy to modify. We use Pi model B with $512 \mathrm{MB}$ of RAM, two USB ports, $100 \mathrm{mb}$ Ethernet port and Debian Wheezy installed shown in Figure 1. We use two types of android devices for client. One is Samsung Galaxy Note II Quad-core 1.6 GHz Cortex-A9 with 2 GB RAM and the other one is Google Nexus 7 Tablet NVIDIA Tegra 3 quad-core processor with 1 GB RAM.

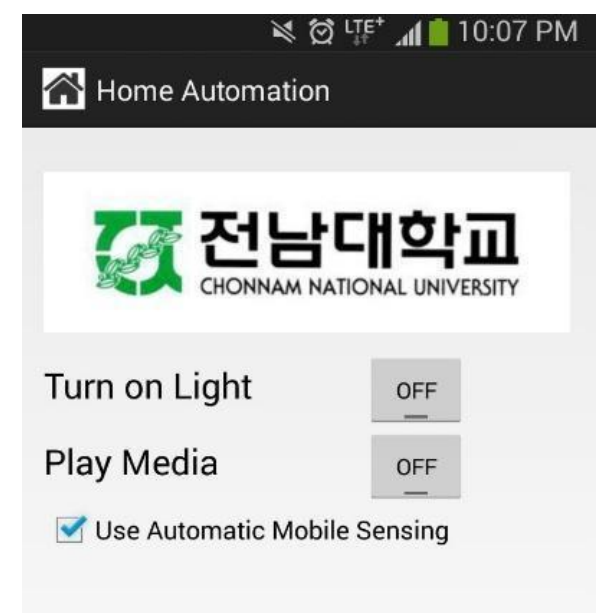

Figure 2. HAS Client Application

\footnotetext{
${ }^{3}$ https://www.indooratlas.com/
} 
In server environment, we implement the command receiver application. In client environment, we implement the application for collecting and processing data sensor. In client application we use Heimcontrol library and modify Heimdroid application. We implement two kinds of action: turn on light and play media. These applications provide the button for each action so the user can control the action manually. To use automatic response based on their daily activity, user can check the checkbox. Then the application will automatically sending the request to the server and get the responses based on user behavior. The GUI of client application can be shown in Figure 2.

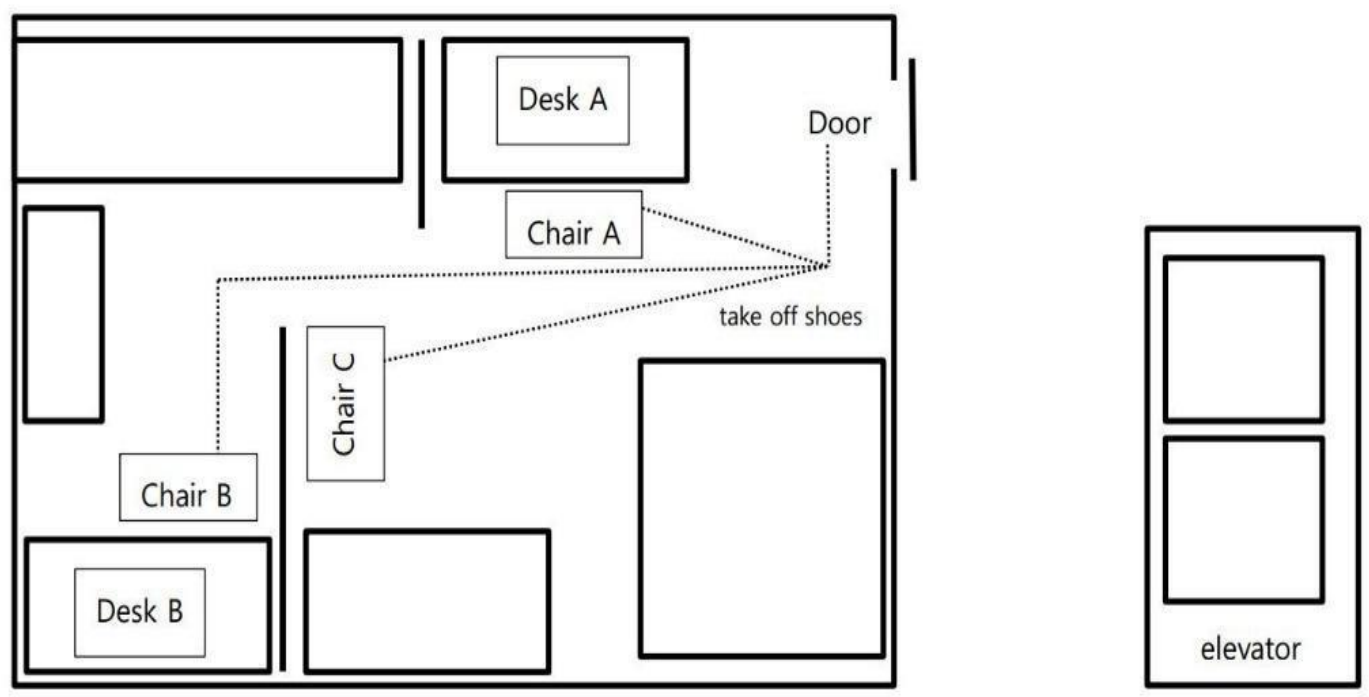

Figure 3. Lab Room 445 4th Floor Engineering Building CNU

\subsection{Scenario of Experiments}

We performed our experiments at our lab's room number 445 4th floor engineering building Chonnam National University (CNU). Figure 3 shows the design of the location (our lab). In this experiment, we have two volunteers: Alice and Bob. More details about their behavior as follows:

- Alice's daily routine

1. He has desk A and chair A.

2. Every day he enters the lab room around 09:00 AM - 11:00 AM.

3. He always use trouser, sometime jeans or others, and he puts his phone in the front hip of his trouser. Figure 4 shows the illustration of this activity.

4. The lab room is on $4^{\text {th }}$ floor. So Alice use elevator from first floor. After enters the lab room, he turns on the light and takes his shoes. Then he sits on his chair.

5. After sits on his chair, he runs the music application and hearing his favorite songs through the headset. After that, he starts working.

- Bob's daily routine

1. He has desk B and chair B.

2. Every day he enters the lab room around 08:00 AM - 09:00 AM. 
3. He has Google Nexus 7's tablet. He always put his tablet on his small side bag. This illustration can be seen in Figure 4. After he enters to the lab room, he turns on the light and takes his shoes then he sits on his chair.

4. After sits on the chair $\mathrm{B}$, he runs the browser application and opens his favorite news portal.

Based on the daily activities above, we implement our approach using following scenario.

1. First scenario, we runs the experiment for 10 days. Every day we ask the volunteers to do their behavior 10 times. So we have 200 data from two volunteers.

2. Second scenario, we runs the experiment for the volunteer to do the other behavior. For this experiment, we ask Alice to do Bob's behavior and vice versa. This experiment also runs 10 times for 10 days. We have 200 data from two volunteers.

3. Third scenario, we ask the volunteers to do their routine behavior but after they enter the lab room they sits on chair $\mathrm{C}$. The location of chair $\mathrm{C}$ can be seen of Figure 3. Again, this experiment run 10 times for 10 days and resulting 200 data from two volunteers.

4. Finally, we have 200 data from each scenario and the total is 600 data.

5. We runs this scenario for two times. First time to collect the data for developing our system (training data). And the second time to prove our approach by counting right and wrong response from our smart home system.

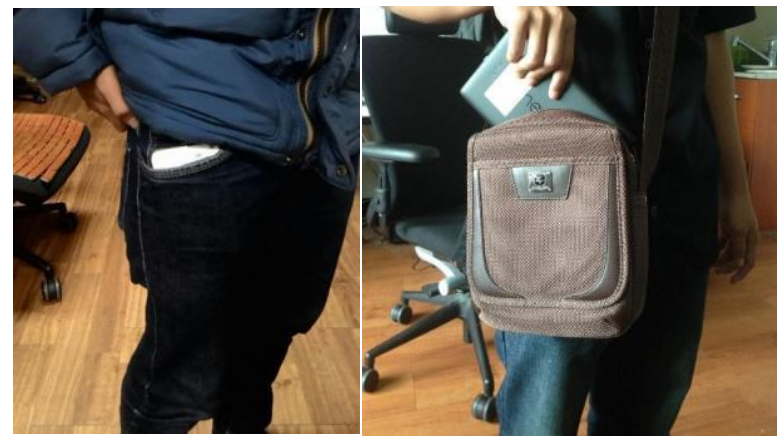

Figure 4. Alice (Left), Bob (Right) and their Smart Phone

\section{Methodologies}

We have 4 type of signals for accelerometer and magnetic field data such as X, Y, Z-axis signal and the magnitude $\mathrm{M}$.

$$
M=\sqrt{a_{X}^{2}+a_{Y}^{2}+a_{Z}^{2}}
$$

Instead of using GPS to know the location of the user, we exploit two of android sensors. First sensor is accelerometer for user identification and activity recognition. The second sensor is magnetic field for define user location inside building. The details of our methodologies can be seen in subsection 4 below.

\subsection{Analyzing Accelerometer Data}

\subsubsection{Time Interpolation}

To process the data sensor, we have to use the same number of values. It is not easy to make the same number of values from data sensor that we collected from user. Therefore, we 
interpolated the acquired signal to $32 \mathrm{~Hz}$ using linear interpolation to ensure that the time interval between two sample-points will be fixed and have same number of values.

\subsubsection{Noise Elimination}

When we collect the data sensor for user walking, there is also some noises. To eliminate the noises, we use Daubechies orthogonal wavelet (Db6). Db6 can be used for signal decomposition and noise reduction. Figure 4 showed the signal after linear interpolation and noise reduction using Db6 at level 3.
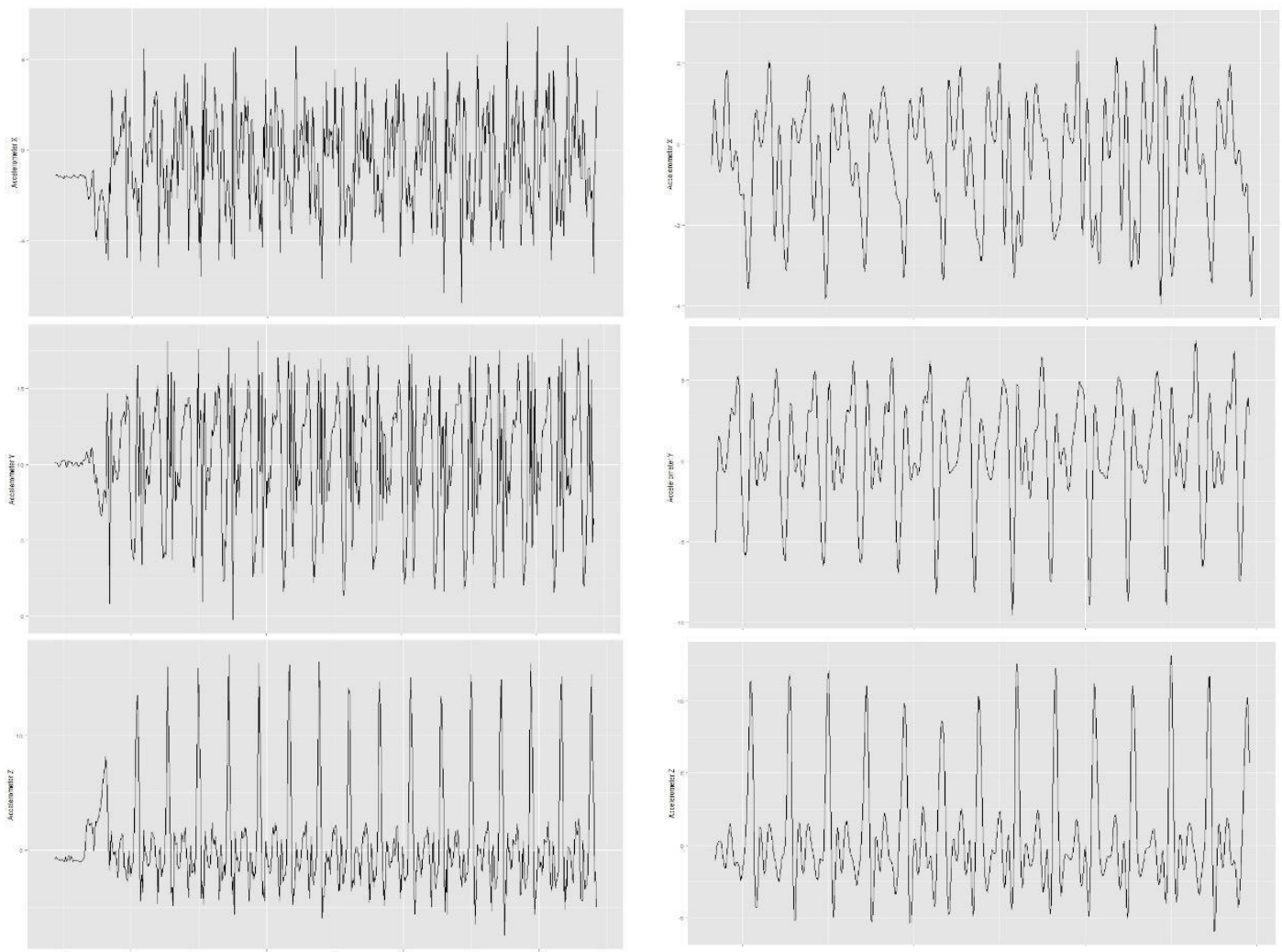

Figure 5. Accelerometer Data Acceleration Before (Left) and After (Right) Interpolation and Noise Reduction

\subsubsection{Gait Cycle Partition}

If we are looking at the Figure 4 that shows the position of the phones for both Alice and Bob, gait characteristics and footsteps are displayed clearly on the Z-dimension. Instead of using the all of 3 accelerometer signals, we only used Z-dimension for gait cycle partition and feature extraction. The last figure in Figure 5 is the plot data from accelerometer $\mathrm{Z}$ signal. Based on Z-signal we saw many of peaks $(P)$ but among the many of peaks $(P)$ we can determine which is the highest peak (TP). The highest peaks among the all peaks are called true peaks (TP). These true peaks can be seen in figure 6 . We designed an algorithm to detect these TPs as follows:

The signal is denoted as $Z(n)$. We extract a set of peaks $P$ from $Z(n)$. A data point is called peak if its value $(\mathrm{m})$ is greater than previous and next one.

$$
\mathrm{P}=\left\{\mathrm{m}_{\mathrm{i}} \mid \mathrm{m}_{\mathrm{i}}>\mathrm{m}_{\mathrm{i}+1} \wedge \mathrm{m}_{\mathrm{i}}>\mathrm{m}_{\mathrm{i}-1}\right\} \text { with } \mathrm{i} \in[1 \ldots \mathrm{n}]
$$


Where $m_{i}$ is the $i^{\text {th }}$ value in $Z(n) . T$ is the threshold to determine TPs using equation (2). The peaks which have value (m) greater than $T$ are identified as set of TPs:

$$
\begin{aligned}
& T=\mu+k \sigma \\
& R=\left\{m \in P \mid m_{i} \geq T\right\}
\end{aligned}
$$

Where $\mu$ is the mean of signal and $\sigma$ is standard deviation of the signal and $k$ is the userdefined constant. Figure 6 shows the threshold T with different $k$ values. In our experiment, choosing $k=\frac{1}{4}$ was the best choice to determine which is peaks and true peaks.

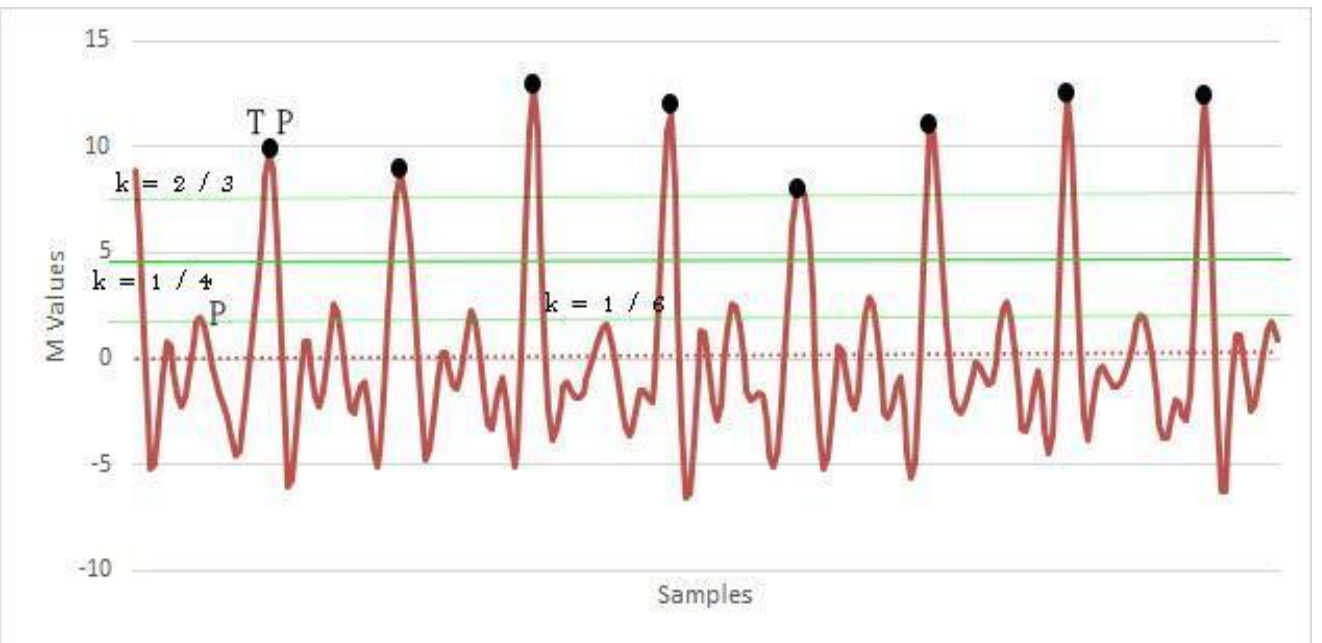

\section{Figure 6. Determine the True Peaks based on the Thresholds T with Various $k$ Values}

\subsubsection{Feature Extraction}

We extract the features in two of domains: time domain and frequency domain. The list of time domain features as follows:

1. Average maximum acceleration for each gait cycles from (X,Y,Z,M signals)

2. Mean acceleration for each gait cycles from (X,Y,Z,M signals)

3. Average minimum acceleration for each gait cycles from (X,Y,Z,M signals)

4. Average absolute different for each gait cycles from (X,Y,Z,M signals)

5. Standard deviation for each gait cycles (X,Y,Z,M signals)

6. Root mean square (RMS) for each gait cycles (X,Y,Z,M signals)

Total of features from time domain are 6 features multiply by 4 signals (X, Y, Z and M). Total are 24 features. We also use Fast Fourier transform (FFT) to transform the data from time domain to frequency domain. We take the first 40 FFT coefficients from each gait signals as the frequency domain features.

\subsection{Analyzing Magnetic Field Data}

To know user location inside building, we use magnetic field sensors. Based on our experiment environment which can be seen in Figure 3, we define five important points: in the front of elevator, open and close the lab's door, Alice's chair, Bob's chair, and C's chair. We collect the data around 100 data from each point. Similar with accelerometer data, we also applied linear interpolation and noise reduction in our dataset. The features were extracted from two types of domains: time domain and frequency domain. The list of features in time domain is same with the features that we use in accelerometer data. For the frequency 
domain, we take first 40 FFT coefficients from all signals as our features. Figure 6 shows the plot of Magnetic field value (M) time domain and frequency domain.

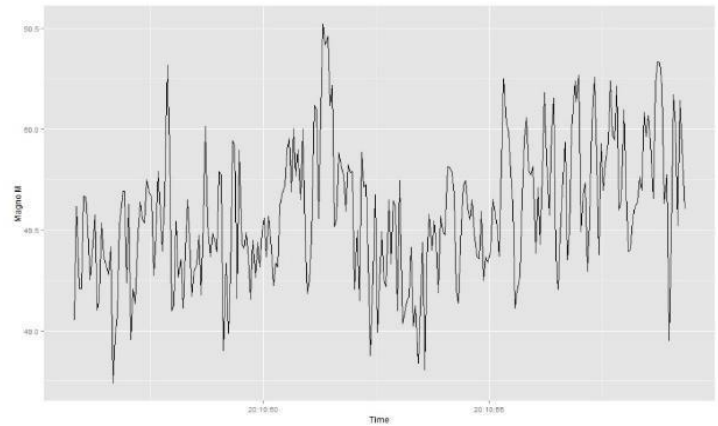

M value of magnetic field from Alice's Chair

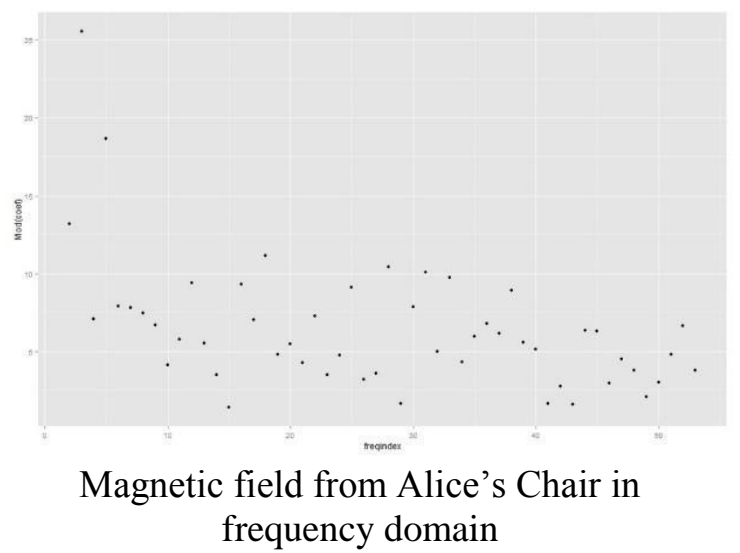

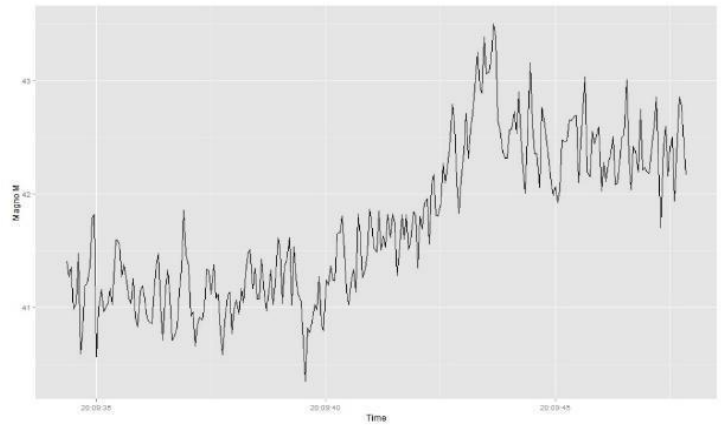

M value of magnetic field from Bob's Chair

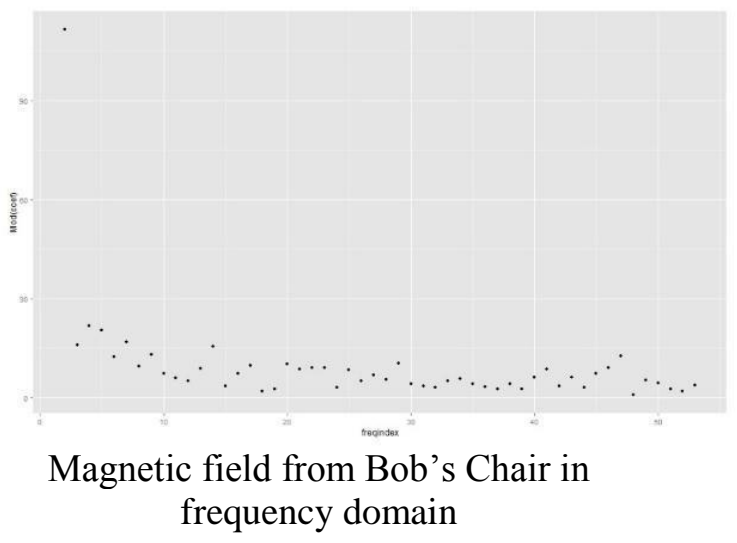

Figure 6. Magnetic Field Signal Plotting in Time Domain and Frequency Domain

\subsection{Classification}

Based on our literature study and our experiments, most of proposed ideas for analyzing accelerometer and magnetic field uses SVM as the method for classification. [22 - 29] uses SVM because this method is good for classification. In this research, we decide to use SVM with RBF kernel. We applied "SVM Tune" to find out the best parameters of the SVM. The best parameters for SVM after we tune are cost: 10 and gamma: 0.5 .

\section{Result and Discussion}

In the previous work [2], we achieved $88 \%$ accuracy in real environment but we have delay problems. Method that we used in previous work was combine features from accelerometer and magnetic field. Based on those features, we applied classification. In this research, our approach are separate the features from accelerometer and magnetic field. We use accelerometer features only for user identification and activity recognition. Magnetic field is use for indoor positioning system. Furthermore, we also use GPS to determine the user location. We can obtain location information of users, which is still in dormitory, on the way go to lab, or already in lab.

This system has three core modules. First is authentication module which is using time and frequency domain features from accelerometer data. Second is activity recognition module which is also using time and frequency domain features from accelerometer data. In this 
module, we only recognize walking, standing, and sitting activities. Third is check position modules. Check position module is module for checking user position inside the building. This module use time and frequency domain features from magnetic field sensor.

Algorithm 5.1 shows the decision making algorithm based on three of main modules in our system. In this system, we have two times of authentication. First authentication is runs when user opens the door. The second authentications reside in the function correctChair. playMedia's function means doing something that is usually done by user. playMedia for Alice is automatically opens the music media player and playing music with his favorite songs. playMedia for Bob is automatically runs the browser application then open his favorite URL news portal.

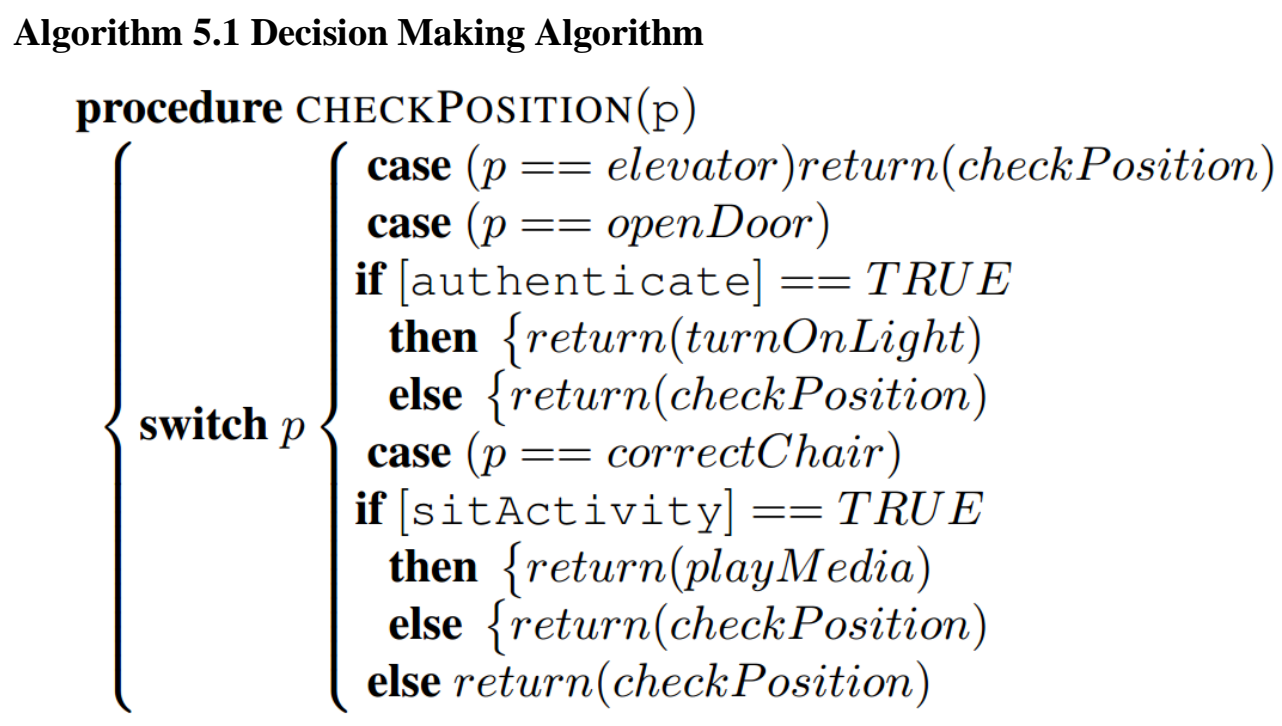

Based on this approach, we achieved $96 \%$ of accuracy. We are analyze this data using PC with $50 \%$ as data training and $50 \%$ as data testing. We achieved $94 \%$ accuracy in real environment with perform 20 times for each scenario but we still get some delay problems. This delay is caused by the data loading and processing time in mobile phone. Searching deeper on our method, we found that our method use many features from the sensors. To reduce the processing time, we use Sequential Forward Selection (SFS) and Sequential Floating Forward Selection (SFFS) to select the best features. We choose SFFS because this algorithm works better than SFS in our dataset. The result of loading time and prediction time for the original method with using SFS and SFFS can be seen in Table 4. Finally, the list features that we use as follows:

- FFT coefficients of accelerometer Z

- FFT coefficients of magnetic field M

- Average absolute distance Z, Y accelerometer

- Average maximum value Z, Y accelerometer

- Average minimum value $\mathrm{Z}, \mathrm{Y}$ accelerometer

Total features that we used are 8 features but with those features, we only achieved $92 \%$ accuracy in real environment. By using this method, we can solve the delay problem. 


\section{Table 4. Comparison of Loading Time and Prediction Time between Original Method and After Applying SFS and SFFS Features Selection}

\begin{tabular}{|l|c|c|}
\hline & Loading Time & Prediction Time \\
\hline Original & $5897 \mathrm{~ms}$ & $1180 \mathrm{~ms}$ \\
SFS & $1099 \mathrm{~ms}$ & $208 \mathrm{~ms}$ \\
SFFS & $823 \mathrm{~ms}$ & $111 \mathrm{~ms}$ \\
\hline
\end{tabular}

\section{Conclusions and Future Work}

This paper shows about defining and evaluating mobile sensing for controlling smart home system. This research shows that we can build and develop smart home system based on user activity. Every human being has own unique behavior and daily routine activities. We can extract the knowledge from that data and build smart home system based on it. This paper also shows that by removing unperformed features, it can decreasing loading time and processing time. This can be solution to avoid delay problem.

In this paper, we use three sensors from mobile phone, GPS for determine user outdoor location, accelerometer sensor for user identification and activity classification, and the last is magnetic field for determine user position inside the building. By using our approach, we achieved $92 \%$ of accuracy in real environment without any delay problem. For future works, we like to improve our system by combining another sensor. Also we can make improve the system to make it smarter. Our current research still using static data for knowledge base. For next research we like to use dynamic data that come from real time sensor data. Real time sensor data will collect user personal data over time and the knowledge system can adapt the dynamic data accordingly. So the responds from our system will be close to the user needs based on his/her current activity.

\section{Acknowledgements}

This research was supported by Basic Science Research Program through the National Research Foundation of Korea (NRF) funded by the Ministry of Education (2012R1A1A2007014).

\section{References}

[1] A. M. Efendi, S. Oh, A. F. P. Negara and D. Choi, "Battery-less 6lowpan-based wireless home automation by use of energy harvesting," International Journal of Distributed Sensor Networks, vol. 2013 (2013).

[2] R. Mafrur, F. Muthohar, K. Bang, D. Lee and D. Choi, "Awareness Home Automation System Based on User Behavior through Mobile Sensing", Computer Science and its Applications Lecture Notes in Electrical Engineering, vol. 330, (2015), pp. 953-959.

[3] U. Sharma and S. Reddy, "Design of home/office automation using wireless sensor network," International Journal of Computer Applications, vol. 43, no. 22, (2012) April.

[4] A.-C. Olteanu, G.-D. Oprina, N. Tapus and S. Zeisberg, "Enabling mobile devices for home automation using zigbee," in 2013 19th International Conference on Control Systems and Computer Science, (2013) May 2931.

[5] D. Basu, G. Moretti, G. S. Gupta and S. Marsland, "Wireless sensor network based smart home: Sensor selection, deployment and monitoring," in Sensors Applications Symposium (SAS) IEEE, (2013) February 19-21.

[6] Q. Sun, W. Yu, N. Kochurov, Q. Hao and F. Hu, "A multi-agent-based intelligent sensor and actuator network design for smart house and home automation," Journal of Sensor and Actuator Networks, (2013).

[7] D. S. Tudose, A. Voinescu, M.-T. Petrareanu, A. Bucur, D. Loghin, A. Bostan and N. Tapus, "Home automation design using 6lowpan wireless sensor networks," in 2011 International Conference on Distributed Computing in Sensor Systems and Workshops (DCOSS), (2011) June 27-29.

[8] M. Kovatsch, M. Weiss and D. Guinard, "Embedding internet technology for home automation," in 2010 IEEE Conference on Emerging Technologies and Factory Automation (ETFA), (2010) September 13-16. 
[9] D.-M. Han and J.-H. Lim, "Design and implementation of smart home energy management systems based on zigbee,” IEEE Transactions, vol. 56, no. 3, (2010).

[10] M. Jahn, M. Jentsch, C. R. Prause, F. Pramudianto, A. Al-Akkad and R. Reiners, "The energy aware smart home," in 2010 5th International Conference on Future Information Technology (FutureTech), (2010) May 21-23.

[11] M. A. Zamora-Izquierdo, J. Santa and A. F. Gomez-Skarmeta, "An integral and networked home automation solution for indoor ambient intelligence," IEEE Pervasive Computing, vol. 9, no. 4, (2010).

[12] J. L. Fernandez, D. P. Losada and E. P. Domonte, "An integrated and low cost home automation system with flexible task scheduling," in XV Workshop of physical agents, (Spain), (2014) June 12.

[13] D. Javale, M. Mohsin, S. Nandanwar and M. Shingate, "Home automation and security system using android adk," International Journal of Electronics Communication and Computer Technology (IJECCT), vol. 3, no. 2, (2013).

[14] M. H. Assaf, R. Mootoo, S. R. Das, E. M. Petriu, V. Groza and S. Biswas, "Sensor based home automation and security system," in 2012 IEEE International Instrumentation and Measurement Technology Conference (I2MTC), (2012) May 13-16.

[15] S. R. Das, S. Chita, N. Peterson, B. A. Shirazi and M. Bhadkamkar, "Home automation and security for mobile devices," in 1st IEEE PerCom Workshop on Pervasive Communities and Service Clouds, (2011) March 21-25.

[16] M. A. Zamora-Izquierdo, J. Santa and A. F. Gomez-Skarmeta, "Hey home, open your door, i'm back! authentication system using ear biometrics for smart home," International Journal of Smart Home, vol. 7, no. 1, (2014).

[17] G. Sankar, K. N. Sreekumar and M. Kumaresan, "Resistive touch screen based home automation system design," Journal of Nano Science and Nao Technology, vol. 2, no. 2, (2013).

[18] S. R. Das, S. Chita, N. Peterson, B. A. Shirazi and M. Bhadkamkar, "Experimental evaluation of speech recognition technologies for voice-based home automation control in a smart home," in 4th Workshop on Speech and Language Processing for Assistive Technologies, (French), (2013) August 21-23.

[19] R. A.Ramlee, M. H. Leong, R. S. S. Singh, M. M. Ismail, M. A. Othman, H. A. Sulaiman, M. H. Misran and M. Said, "Bluetooth remote home automation system using android application," The International Journal of Engineering And Science (IJES), vol. 2, no. 1, (2014).

[20] R. Piyare and M. Tazil, "Bluetooth based home automation system using cell phone," in 2011 IEEE 15th International Symposium on Consumer Electronics, (2014) June 14-17.

[21] S. Jain, A. Vaibhav and L. Goyal, "Raspberry pi based interactive home automation system through e-mail," in 2014 International Conference on Reliability, Optimization and Information Technology, (2014) February 6-8.

[22] A. Primo, V. V. Phoha, R. Kumar and A. Serwadda, "Context-aware active authentication using smartphone accelerometer measurements," in CVPR 2014 workshop, (2014).

[23] F. Juefei-Xu, C. Bhagavatula, A. Jaech, U. Prasad and M. Savvides, "Gait-id on the move: Pace independent human identification using cell phone accelerometer dynamics," in IEEE, (2012).

[24] C. Nickel and C. Busch, "Classifying accelerometer data via hidden markov models to authenticate people by the way they walk," in 2011 IEEE International Carnahan Conference on Security Technology (ICCST), (2011).

[25] S. Sprager and D. Zazula, "A cumulant-based method for gait identification using accelerometer data with principal component analysis and support vector machine," Journal WSEAS Transactions on Signal Processing, vol. 5, no. 11, (2009).

[26] M. O. Derawi, C. Nickel, P. Bours, and C. Busch, "Unobtrusive user-authentication on mobile phones using biometric gait recoginition," in 6th IIH-MSP, (2010).

[27] J. Frank, S. Mannor and D. Precup, "Activity and gait recognition with time-delay embeddings," in Proceedings of the Twenty-Fourth AAAI Conference on Artificial Intelligence (AAAI-10), (2010).

[28] C. Nickel and C. Busch, "Classifying accelerometer data via hidden markov models to authenticate people by the way they walk," in 2011 IEEE ICCST, (2011).

[29] Y. Kwon, K. Kang and C. Bae, "Unsupervised learning for human activity recognition using smartphone sensors," Journal Expert Systems with Applications, vol. 41, (2014).

[30] O. Banos, M. Damas, H. Pomares, A. Prieto and I. Rojas, "Daily living activity recog- nition based on statistical feature quality group selection," Journal Expert Systems with Applications, vol. 39, (2012).

[31] A. M. Khan, A. Tufail, A. M. Khattak and T. H.Laine, "Activity recognition on smart- phones via sensorfusion and kda-based svms," International Journal of Distributed Sensor Networks, vol. 2014, (2014).

[32] M. A. Ayu, T. Mantoro, A. F. A. Matin and S. S. O. Basamh, "Recognizing user activity based on accelerometer data from a mobile phone," in 2011 IEEE Symposium on Computers Informatics, (2011).

[33] C. E. an Tejada, J. C. Carrasco-Jimenez and R. Brena, "Location identification using a magnetic-field-based fft signature," in ANT 2013, (2013). 
[34] K. Yun, Y. Jo, N. Kim, U. Jo and Y. Kim, "Experimental performance evaluation of a sim- ple indoor positioning scheme with two commonly used sensors," in SERSC proceedings, (2013).

[35] B. Li, T. Gallagher, C. Rizos and A. G. Dempster, "Using geomagnetic field for indoor positioning," in International Global Navigation Satellite Systems Society IGNSS Symposium 2013, (Australia), (2013).

[36] C. E. Galvan-Tejada, J. P. Garca-Vazquez and R. F. Brena, "Magnetic field feature extraction and selection for indoor location estimation,” Sensors (ISSN 1424-8220; CODEN: SENSC9), vol. 2014, (2014).

[37] N. L. Dortz, F. Gain and P. Zetterberg, "Wifi fingerprint indoor positioning system using probability distribution comparison," in ICASSP, vol. 2012, (2012).

[38] Y. Chen, D. Lymberopoulos, J. Liu and B. Priyantha, "Fm-based indoor localization," in ACM Proceeding MobiSys, vol. 2012, (2012).
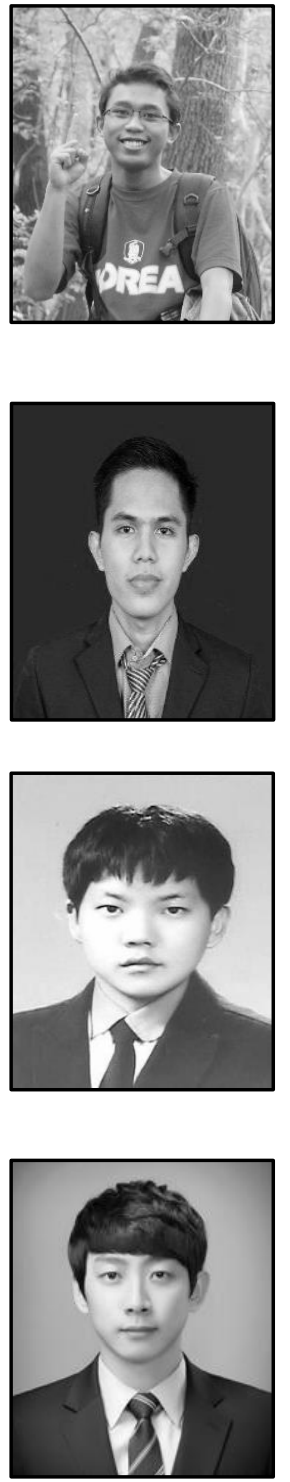

\section{Authors}

Rischan Mafrur, He received the B.Eng in Computer Engineering from Sunan Kalijaga State Islamic University Indonesia in 2013. Since September 2013, he has been with the Network Systems Lab, Chonnam National University, Gwangju, Korea, pursuing a Master degree in Electronics \& Computer Engineering. His main research interests include ubiquitous computing, data processing and analysis, data mining and web mining.

Priagung Khusumanegara, He received the B.Eng in Computer Engineering from Universitas Indonesia in early 2014. Since September 2014, he has been with the Network Systems Lab, Chonnam National University, Gwangju, Korea, pursuing a Master degree in Electronics \& Computer Engineering.

Kihyun Bang, He received Engineering degree in Faculty of Life Science and Technology, Chonnam National University in 2013. He is currently studying for his MS Degree in School of Electronics and Computer Engineering, Chonnam National University, South Korea. His research interests are computer network, software defined networking and ubiquitous healthcare.

Dokyeong Lee, He received the B.Eng in Information \& Communication Engineering from Honam University in early 2013. Since 2013, he has been with the Network Systems Lab, Chonnam National University, Gwangju, Korea, pursuing a Master degree in Electronics \& Computer Engineering. His main research interests include sensor network development and internet of things 


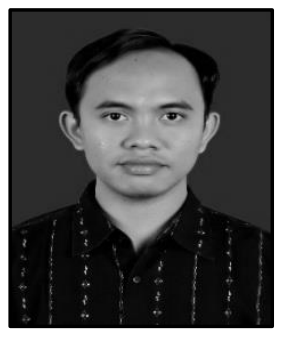

I Gde Dharma Nugraha, $\mathrm{He}$ receive the B.Eng in Electrical Engineering and Master Degree in Computer Engineering from Universitas Indonesia. Since September 2014, he has been with the Network System Lab, Chonnam National University, Gwangju, Korea pursuing doctoral degree in Electrical \& Computer Engineering.

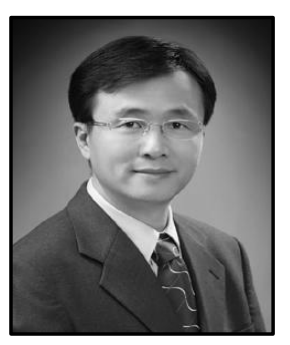

Deokjai Choi, He received the B.S., M.S in Computer Science from Seoul National University, Korea in 1982 and from KAIST 1984 respectively and also received Ph.D. in Computer Science and telecommunication from University of Missouri-Kansas City, USA in 1995. Since 1996 until now, he has been serving as Professor in School of Electronics and Computer Engineering, Chonnam National University, Korea. 